\title{
Lumière et mélatonine pour la maîtrise de la reproduction des ovins et des caprins
}

\author{
P Chemineau, B Malpaux, Y Guérin, \\ F Maurice, A Daveau, J Pelletier
}

INRA, physiologie de la reproduction, 37380 Nouzilly, France

(Reçu le 10 octobre1991; accepté le 15 janvier 1992)

\begin{abstract}
Résumé - Les traitements utilisant la succession "JL"-jours décroissants ou "JL"-mélatonine sont très efficaces pour avancer la puberté chez les jeunes béliers mis en testage sur descendance par insémination artificielle (IA). Chez les adultes, les mêmes traitements produisent une augmentation importante du poids testiculaire. Chez la chèvre, la succession " $\mathrm{JL}$ "-mélatonine induit et maintient très efficacement cestrus et ovulation au printemps, conduisant à une fertilité élevée en lutte naturelle, inhabituelle à cette période de l'année. Chez les races saisonnées de brebis du Nord de l'Europe, un tel traitement apparaît moins efficace que chez les caprins. La mélatonine seule peut être utilisée; elle provoque une avance de saison d'un mois à un mois et demi environ, et accroit la fécondité des brebis $(+0,2 \mathrm{a} \pm 0,4$ agneau par brebis). L'alternance mensuelle de $\mathrm{JL}$ et $\mathrm{JC}$ abolit les variations saisonnières du comportement sexuel et de l'activité spermatogénétique, ce qui accroît considérablement la production spermatique quantitative et qualitative dans les deux espèces. Des mâles ainsi traités peuvent être utilisés tout au long de l'année pour produire des doses d'IA sans variation de la qualité de la semence ni de la fertilité. Des rythmes accélérés peuvent désormais être aussi utilisés en bâtiment ouvert en alternant des "JL" et la mélatonine chez des béliers maintenus dans des conditions traditionnelles d'élevage. En revanche, chez la brebis, de tels rythmes ne permettent pas d'abolir les variations saisonnières d'activité ovulatoire.
\end{abstract}

\section{photopériode / mélatonine / saison / insémination artificielle / ovin / caprin}

Summary - Light and melatonin for the control of sheep and goat reproduction. Breeds of sheep and goats trom temperate latitudes present seasonal variations in breeding activity which limit their productivity. These variations are controlled by annual photoperiodic changes. Short days (SD) are stimulatory to sexual activity, but, if used for a long time, they cause the appearance of refractoriness which stops reproductive activity. Refractoriness can be "broken" by exposing the animals to the opposite photoperiod ie long days, (LD), leading to the principle that alternations between $L D$ and $S D$ are essential for the photoperiodic control of seasonal reproduction. The interruption of night by light can mimic an $L D$ ("LD") and melatonin treatments can mimic an SD ("SD"). All these observations are important for practical applications. For out-of-season control of sexual activity, treatments using the succession " $L D$ "-decreasing days or " $L D$ "-melatonin were demonstrated to be very efficient in advan-cing puberty in young rams. They caused a dramatic increase in sperm production, allowing earlier use of these animals in progeny tests for artificial insemination (Al). In adult rams, such treatments also caused an important increase in testicular weight (significantly different from control rams for $100 \mathrm{~d}$ in the spring). In the female goat, the succession "LD"-melatonin is very efficient in inducing and maintaining oestrous and ovulatory activities in spring, leading to an unusually 
high fertility after natural mating during this period of the year. In the seasonal breeds of ewes from Northern Europe, such a treatment has so far appeared less efficient than in she-goats. However, melatonin alone can be used after the end of May to advance sexual season and increase fecundity. Induction of permanent reproductive activity in rams and he-goats was made possible by the obsenvation that monthly alternations between $L D$ and $S D$ (= short light cycles) abolished seasonality of behavioural and spermatogenetic activities. Such treatment greatly improved quantitative and qualitative sperm production in both species. The males could be used all year round to produce Al doses without variations in sperm quality and no alteration in fertility. Short light cycles can be used in open barns by alternating " $L D$ " and melatonin which abolishes seasonal testicular weight variations. This last result, $o b$ tained in lle-de-France rams, is the first record of induction of a permanently high sexual activity in normally seasonal males kept under normal husbandry conditions. On the contrary, in the ewe, such short light cycles were unable to abolish seasonality of ovulatory activity. The knowledge of the different effects of photoperiod on the neuroendocrine pathways and the reproductive activity in sheep and goats has therefore allowed light treatments to be successfully tested to control seasonal reproductive activity under field conditions and in males raised in Al centres.

photoperiod / melatonin / reproduction / sheep / goat

\section{INTRODUCTION}

L'activité reproductrice de la plupart des animaux domestiques originaires des zones tempérées présente des variations saisonnières. Celles-ci sont plus ou moins marquées selon les espèces; les petits ruminants manifestant des périodes d'arrêt complet de leur reproduction (Ortavant et al, 1985).

Chez les ovins/caprins, espèces dites de "jours courts", la lumière (par le fait des variations de la durée de la phase claire du jour, la photopériode), constitue certainement la principale cause des importantes variations saisonnières de reproduction observées pour l'ensemble des races d'Europe du Nord. Des résultats récents permettent, d'une part de mieux connaître et de mieux comprendre les mécanismes par lesquels la photopériode agit sur la reproduction et d'autre part, de mettre au point des manipulations lumineuses ou des traitements biochimiques, aboutissant au report ou à l'annulation des variations saisonnières.

Étudiés depuis de nombreuses années chez les espèces présentant une sensibilité marquée, les effets directs de la photo- période sont assez bien connus maintenant. Cependant, les mécanismes impliqués dans les effets de la lumière sur la reproduction ne sont pas encore tous élucidés. Certains progrès récents permettent néanmoins d'envisager, chez les petits ruminants, la mise au point de traitements pour maîtriser les variations saisonnières de reproduction.

\section{EFFETS DES VARIATIONS PHOTOPÉRIODIQUES SUR LA REPRODUCTION DES PETITS RUMINANTS}

\section{Effets des jours courts (JC) et des traitements de type «jours courts"(«JC»)}

Les JC (dans la plupart des cas, < 12/24 h de lumière succédant à des jours longs, $J L$ ), appliqués pendant suffisamment longtemps ont un effet stimulateur sur la reproduction. Les ovulations débutent environ $80 \mathrm{j}$ chez la chèvre alpine et $50 \mathrm{j}$ chez la brebis lle-de-France, après le changement JLJJC (Chemineau et al, 1988). Cependant ces JC ne sont pas toujours stimulateurs, 
puisqu'après $70 \mathrm{j}$ d'activité sexuelle, celleci s'arrête et les animaux deviennent alors «réfractaires» aux JC. C'est l'installation de cet état en hiver qui est responsable, en partie au moins, de l'arrêt saisonnier de l'activité aux alentours du mois de février (Robinson et Karsch, 1984).

Les JC peuvent être remplacés par un traitement à la mélatonine (Kennaway et al, 1980; Kennaway et al, 1982; Arendt et al, 1983). Ceci est vérifié même si les animaux sont maintenus en JL (si leurs yeux perçoivent des $J L$ ), ce qui est généralement le cas au printemps et en été lorsque l'on souhaite induire une activité sexuelle à contre-saison, et qu'il est impossible de maintenir les animaux dans un local fermé. L'utilisation est donc envisageable en bâtiment ouvert ou au pâturage, les animaux réagissent au traitement comme aux JC. La mélatonine peut être distribuée dans l'alimentation quotidienne en fin d'après midi (ce qui correspondrait au crépuscule des JC) ou bien, et c'est la forme la plus facile à utiliser, libérée en permanence par un dispositif sous-cutané (implant) ou intraruminal (bolus).

II faut également remarquer que les JC et/ou la mélatonine, chez les brebis et chez les chèvres, ont un effet dépresseur sur la lactation.

\section{Effets des jours longs (JL) et des traitements de type jours «longs" ( $J L ")$}

Les $\mathrm{JL}$ (dans la plupart des cas $>12 \mathrm{~h}$ de lumière/24 $\mathrm{h}$, succédant à des JC), appliqués pendant suffisamment longtemps, inhibent la reproduction. La chèvre alpine arrête ses ovulations environ 80 j et la brebis lle-de-France environ $35 \mathrm{j}$ après le passage JC/JL. Cependant ces JL ne demeurent pas toujours inhibiteurs; après un certain temps, l'activité sexuelle reprend; les animaux deviennent alors "réfractaires» aux JL (Robinson et al, 1985). L'installation d'un état réfractaire aux JL est, en partie au moins, probablement responsable du déclenchement du début de la saison sexuelle en août-septembre. Mais les $\mathrm{JL}$ ont également la propriété de «lever» l'état réfractaire aux $\mathrm{JC}$, et donc de leur restituer un effet stimulateur ce qui est intéressant pour débuter les traitements en fin d'hiver-printemps.

II est possible de remplacer ces JL par un traitement photopériodique de type "لL" plus économe en électricité. Le moment d'éclairement pendant le rythme circadien (au cours de 24 h) est en effet aussi important que sa durée elle-même. En effet, lorsque la lumière est présente de 15 à $18 \mathrm{~h}$ après l'aube, les animaux font la lecture d'un jour long (Ortavant, 1977; Pelletier et al, 1981; Pelletier et Thimonier, 1987). Cette propriété permet d'utiliser dans des bâtiments normaux, une courte période d'éclairement pendant la nuit après avoir réalisé une aube fixe, au lieu de JL réels, plus coûteux en énergie. Ce traitement est donc appelé de type «JL» ou également «flash» bien que la durée d'éclairement nocturne soit de 1 à $2 \mathrm{~h}$.

\section{Importance du "passé photopériodique", existence d'un rythme endogène}

Les effets de la photopériode mentionnés ci-dessus ont été identifiés dans des bâtiments fermés où la lumière est strictement contrôlée. Dans les conditions naturelles, d'autres phénomènes contribuent aux variations saisonnières de reproduction.

Le «passé photopériodique» des animaux conditionne leur réponse aux changements. Ainsi, $12 \mathrm{~h}$ de lumière/j seront considérées comme des JC si les animaux étaient auparavant placés sous une photopériode de $16 \mathrm{~h}$ de lumière/j, mais comme 
des JL si ceux-ci percevaient auparavant $8 \mathrm{~h}$ de lumière/j .

II est également bien admis maintenant que la plupart des mammifères possèdent un rythme endogène de reproduction qui ne se manifeste, de façon évidente, que lors de l'absence totale de variations photopériodiques. Sa période serait proche de 10-11 mois, chez le mâle (Howles et al, 1980) et la femelle (Thimonier, 1989). Les stimulations lumineuses auraient alors pour rôle de synchroniser ce rythme endogène, pour lui imposer une période annuelle et que la reproduction ait lieu au moment où l'environnement naturel est le plus favorable à la survie des jeunes.

Les tentatives les plus récentes de "reconstruction" des effets de la photopériode en conditions naturelles laissent penser que tous les phénomènes précédemment décrits interviennent au cours de l'année (Malpaux et al, 1989; Chemineau et al, 1989); leur importance relative, leur succession temporelle ainsi que leur intervention dans différentes races restent toutefois largement inconnues.

\section{MÉCANISMES MIS EN JEU PAR LES VARIATIONS PHOTOPÉRIODIQUES}

L'information photopériodique est transmise par l'intermédiaire de la rétine, jusqu'à la glande pinéale (appelée aussi épiphyse) par voie nerveuse. Le tracé nerveux passe par différents noyaux hypothalamiques (suprachiasmatique et paraventriculaire, entre autres), puis par le ganglion cervical supérieur, situé dans le cou. L'ablation de ce ganglion empêche toute interprétation du signal photopériodique.

La perception de l'obscurité par des récepteurs adrénergiques au niveau des pinéalocytes (cellules sécrétrices de la pi- néale), entraîne la synthèse et la libération de la mélatonine, considérée actuellement comme le messager permettant au système nerveux central d'interpréter le signal photopériodique. La mélatonine n'est, en effet, synthétisée et sécrétée que pendant la nuit (fig 1).

Lorsque les nuits sont courtes (jours longs), la durée de sécrétion de mélatonine est courte; au contraire, en jours courts, (nuits longues), la durée de sécrétion de mélatonine est longue. C'est par la durée de cette sécrétion que les animaux connaissent la durée du jour. Ainsi, la perfusion de mélatonine à des brebis pinéalectomisées produit des effets variables selon la durée de perfusion : une longue durée par $24 \mathrm{~h}$ provoque un déclenchement de leur activité sexuelle et inversement.

S'il est donc connu que la mélatonine est le messager intermédiaire entre l'information photopériodique et les cellules à $\mathrm{LH}-\mathrm{RH}$ de l'hypothalamus, plusieurs "chaînons" sont encore manquants. Les sites récepteurs du système nerveux central et le mode d'action précis de la mélatonine ne sont toujours pas identifiés avec certitude. Des travaux récents, chez la brebis et le bélier, mettent en évidence la présence de sites d'action dans l'hypothalamus médio-basal (Malpaux et al, 1990) ou de récepteurs dans la tige hypophysaire (de Reviers et al, 1989), dont les rôles dans ces parties du cerveau restent encore très mal connus.

In fine, c'est donc l'activité des cellules sécrétrices de $\mathrm{LH}-\mathrm{RH}$ qui se trouve stimulée ou inhibée. Un certain temps après le passage sous une photopériode stimulatrice, leur activité de décharge pulsatile augmente fortement, ce qui entraîne l'accroissement de la fréquence des «pulses» de LH par l'hypohyse, puis la stimulation gonadique et enfin la reprise de l'activité sexuelle. 


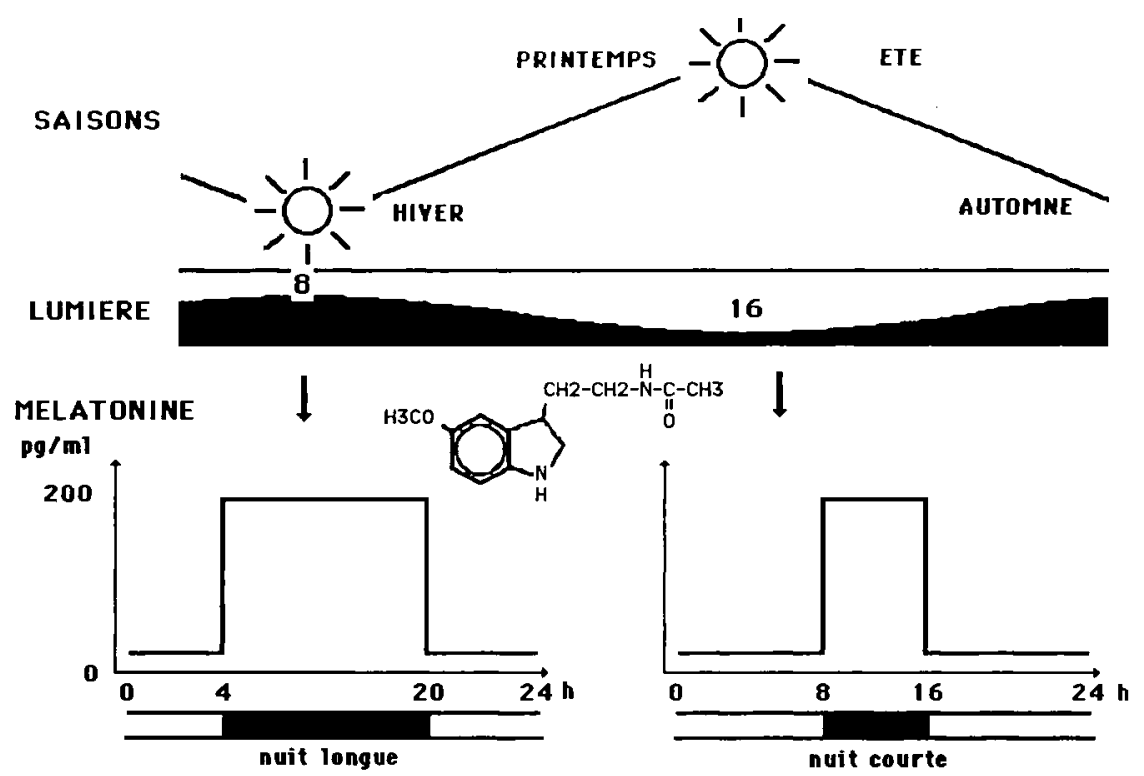

Fig 1. Représentation schématique des changements dans la concentration de mélatonine plasmatique selon la saison et la durée du jour.

\section{MANIPULATIONS PHOTOPÉRIODIQUES PERMETTANT LE CONTRÓLE DE LA REPRODUCTION CHEZ LES PETITS RUMINANTS}

Les traitements photopériodiques actuellement expérimentés en vue du contrôle de l'activité sexuelle à contre-saison des ovins et des caprins, sont directement dérivés des concepts exposés ci-dessus. Ils poursuivent principalement 3 objectifs :

- l'induction et le maintien à contre-saison d'une activité spermatogénétique élevée chez le mâle et de la cyclicité chez la femelle, les traitements hormonaux "classiques" actuels, ne permettant l'induction que d'une seule période d'ovulation, sans retours en chaleurs;

- l'abolition totale des variations saisonnières d'activité sexuelle, surtout intéressante chez les mâles.
- l'avance de la date de la saison sexuelle chez la femelle.

\section{Induction et maintien \\ d'une activité sexuelle à contre-saison}

Le principe retenu pour ce type de traitement est la succession d'une période de «JL» pendant l'hiver et d'une période de «jours courts» ou décroissants (fig 2) pendant le printemps, afin d'aboutir à une activité sexuelle en fin de printemps début d'été.

\section{Essais réalisés chez les mâles}

Les essais ont concerné des jeunes béliers nés à l'automne et des béliers adultes, essentiellement dans des centres d'IA souhaitant disposer de semence fraîche en grande quantité et de bonne 
Bâtiments fermés:
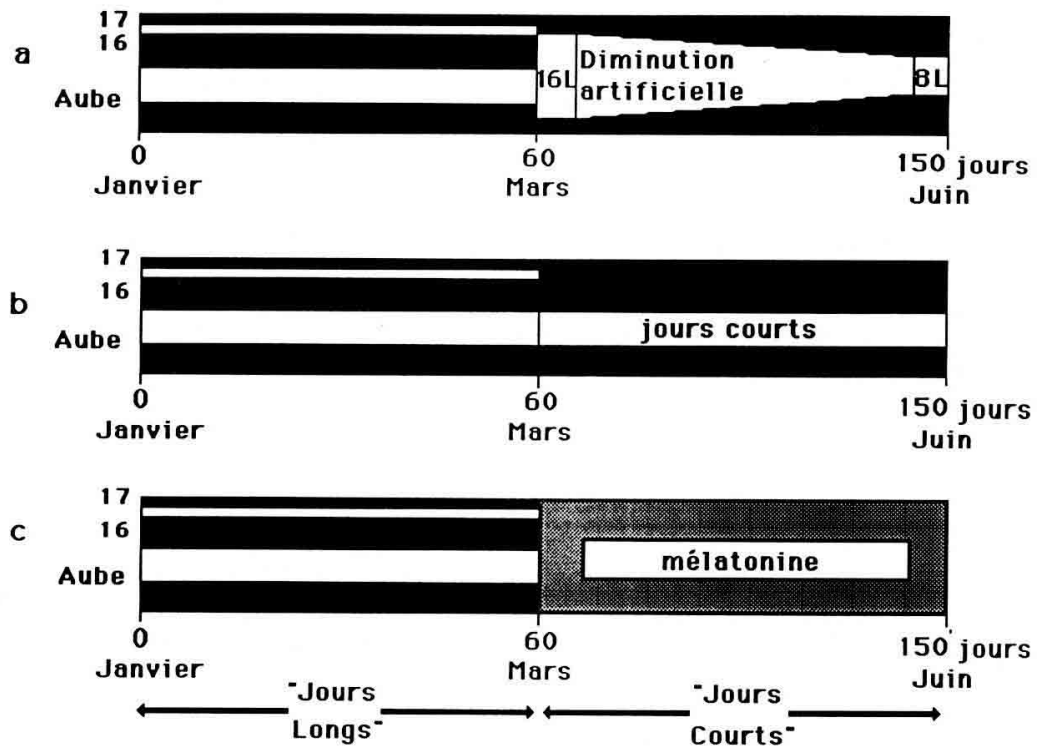

Bâtiments ouverts:

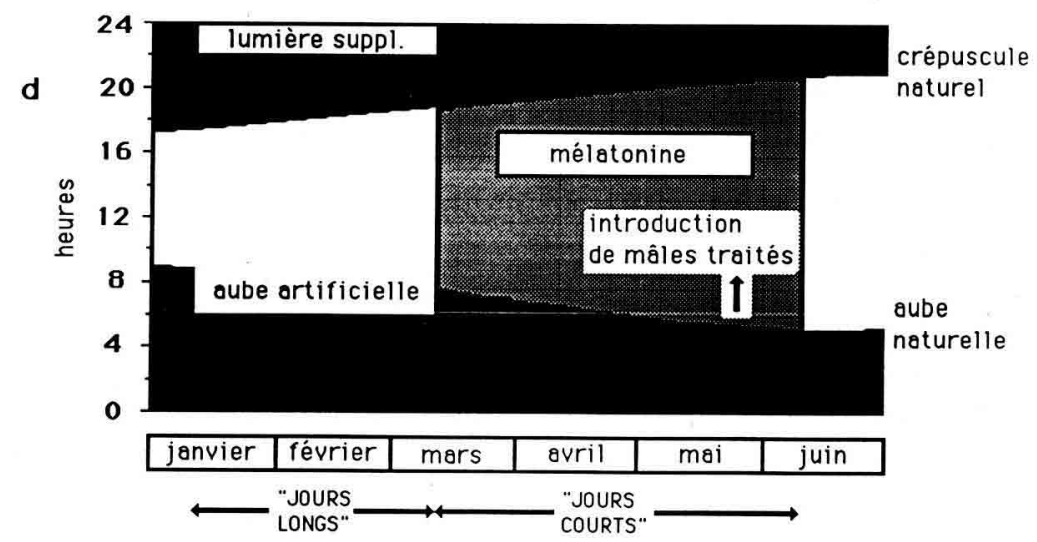

Fig 2. Traitements photopériodiques utilisables en bâtiments fermés ou en bâtiments ouverts.

qualité, alors que les mâles producteurs sont habituellement en pleine contresaison. Les traitements expérimentés permettent d'avancer la puberté des jeunes béliers (Colas et al, 1987) et d'obtenir plus de doses de semence à mettre en place pour le testage sur descendance (tableau 1). L'efficacité de ces traitements est telle que 2 des plus grands centres d'IA français (bassin de Roquefort) ont désormais 
Tableau I. Production spermatique chez des agneaux Lacaune subissant un traitement photopériodique de deux mois de "JL" suivis de trois mois de jours décroissants (Centres d'I.A. du Rayon de Roquefort area, Ovitest et Conféderation des Producteurs de Roquefort).

\begin{tabular}{|c|c|c|c|}
\hline & $\begin{array}{l}\text { Agneaux } \\
\text { Témoins }\end{array}$ & & $\begin{array}{l}\text { Agneaux } \\
\text { traités }\end{array}$ \\
\hline $\begin{array}{l}\text { Nombre d'agneaux } \\
\text { Nombre d'agneaux collectés avec succès } \\
\text { la } 1^{\text {re }} \text { année }(\%)\end{array}$ & $\begin{array}{c}43 \\
33(77)\end{array}$ & NS & $\begin{array}{c}53 \\
41(77)\end{array}$ \\
\hline $\begin{array}{l}\text { Nombre total de doses d'IA par bélier collecté * } \\
\text { Centre } 1: \text { (total période de collecte) } \\
\text { Centre } 2: \text { (par éjaculat) }\end{array}$ & $\begin{array}{l}29,5 \pm 31,8 \\
11,0 \pm 0,5\end{array}$ & $\begin{array}{l}p<0,05 \\
p<0,001\end{array}$ & $\begin{array}{r}63,3 \pm 36,4 \\
14,2 \pm 0,7\end{array}$ \\
\hline
\end{tabular}

$400 \times 10^{6} \mathrm{spz}$, par dose $(\mathrm{m} \pm \mathrm{sem})$.

décidé de traiter tous les agneaux du schéma de sélection laitier.

Chez les adultes, la succession «JL» puis «JC» (réels ou simulés par la pose d'implants de mélatonine) ou jours décroissants, permet d'obtenir une reprise importante de l'activité spermatogénétique et la production d'une semence utilisable pour I'IA (fig 3). Ce type de traitement est ac- tuellement en cours d'essai dans plusieurs centres d'IA français.

\section{Essais réalisés chez les femelles}

Le schéma expérimenté est celui utilisant la succession dite «flash-mélatonine» (fig 2).

Dans l'espèce caprine, les résultats montrent très clairement la nécessité d'uti-

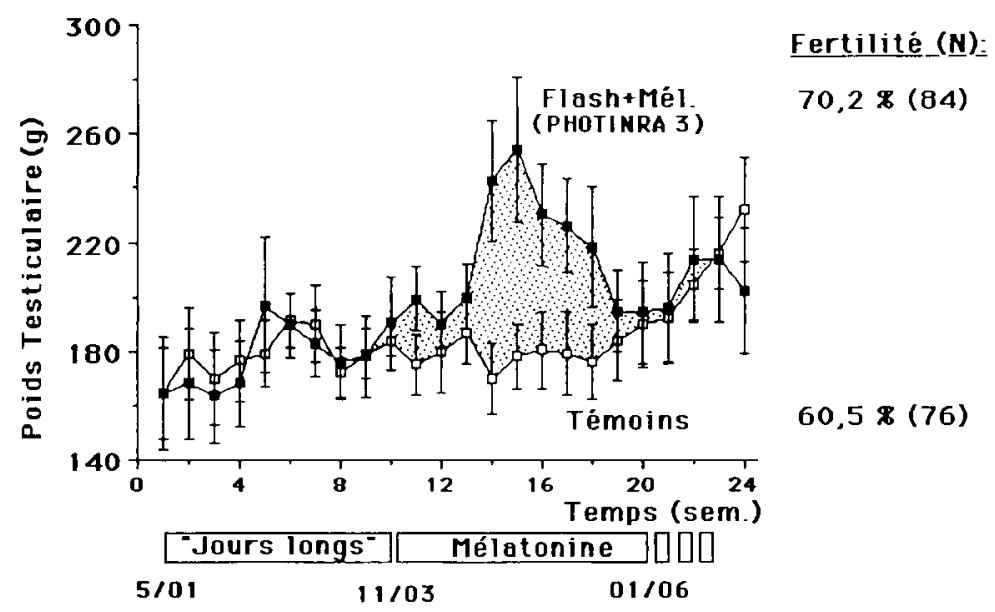

Fig 3. Poids testiculaire et fertilité chez des béliers lle-de-France adultes témoins traités par la succession “jours longs" - implants de mélatonine (Guérin Y, 1988, résultats non publiés). 
liser la succession de ces 2 parties du traitement pour aboutir à une activité sexuelle maximale au printemps (Chemineau et al, 1986; Chemineau, 1989) (tableau II). Dans ces conditions, en utilisant des boucs traités de la même façon et utilisés en lutte naturelle, la fertilité et la prolificité sont très proches, voire identiques à celles de la saison sexuelle annuelle, alors que les femelles sont fécondées en avril-mai. Le traitement permet d'obtenir une meilleure réponse à l'«effet bouc», mais surtout d'aboutir à une cyclicité ovulatoire et œstrienne qui va conditionner les bons résultats de fertilité.

L'effet du mode de distribution de la mélatonine a également été testé chez les caprins en comparant une distribution quotidienne dans l'après-midi par injection ou ingestion, avec l'insertion d'implants souscutanés délivrant une quantité constante. Les 3 modes de distribution ont sensiblement la même efficacité (Chemineau et al, 1988) (fig 4).
Chez la brebis, il semble que l'obtention d'une activité cyclique à contre-saison soit plus délicate. Actuellement, avec les races utilisées, l'effet bélier seul (même avec des béliers traités de la même façon) ne suffit pas pour induire des ovulations; le recours aux traitements hormonaux "classiques" reste nécessaire. Une fois induites, toutefois, ces ovulations se renouvellent ce qui n'est pas le cas chez les animaux témoins. II est généralement possible d'obtenir une meilleure fertilité à l'œstrus induit associée à des fécondations sur retours, ce qui entraîne une bonne fertilité totale de la lutte de contre-saison.

\section{Abolition totale des variations saisonnières}

L'application des différents concepts exposés dans la première partie et notamment celui concernant la nécessité d'une alternance entre photopériodes opposées pour éviter l'installation d'un état réfractaire, a

Tableau II . Mise en évidence de la nécessité d'utiliser la succession lumière + mélatonine, pour l'obtention d'une activité sexuelle et d'une fertilité maximale, à contre-saison chez la chèvre laitière française.

\begin{tabular}{|c|c|c|c|c|}
\hline & \multicolumn{4}{|c|}{ Lots expérimentaux } \\
\hline & Témoins & Mélatonine & Lumière & $\begin{array}{c}\text { Lumière + } \\
\text { Mélatonine }\end{array}$ \\
\hline \multicolumn{5}{|l|}{ Expérience $1^{\mathrm{a}}$} \\
\hline Effectif & 8 & 8 & 8 & 8 \\
\hline Femelles ovulant encore 2,5 mois après le bouc & 0 & 1 & 0 & 6 \\
\hline Femelles venues au moins 2 fois en chaleurs & 2 & 5 & 5 & 8 \\
\hline \multicolumn{5}{|l|}{ Expérience $2^{\mathrm{b}}$} \\
\hline Effectif & 10 & 28 & 8 & 44 \\
\hline Proportion ovulant pendant la lutte (\%) & 10 & 46 & 63 & 91 \\
\hline Fertilité des femelles en \% Mise Bas & 10 & 39 & 63 & 86 \\
\hline Date moyenne de fécondation & $25 / 04$ & $28 / 04$ & $03 / 05$ & $27 / 04$ \\
\hline
\end{tabular}

a Adultes Saanen en lait, boucs vasectomisés du 30 mai au 30 juin; ${ }^{b}$ jeunes Alpines d'un an, boucs entiers du 15 avril au 27 mai. 


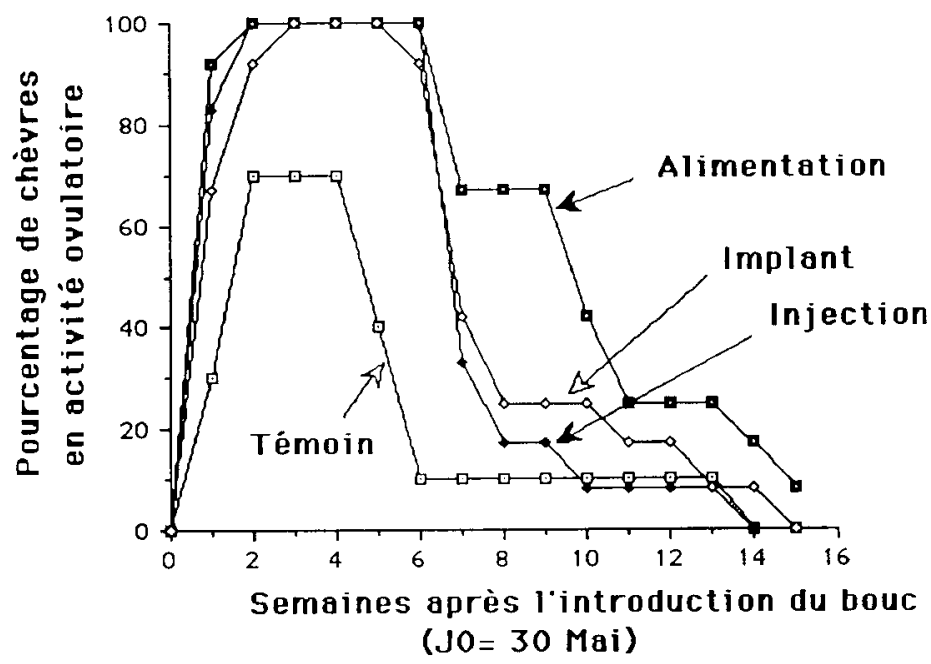

Fig 4. Durée de l'activité ovulatoire induite chez les chèvres témoins ou traitées par la succession “jours longs" - mélatonine (d'après Chemineau et al, 1988).

permis d'envisager un traitement susceptible d'abolir les variations saisonnières de la reproduction chez le bélier. Testé avec succès dans cette espèce, ce schéma a également été appliqué au bouc.

\section{Chez le bélier}

L'alternance très rapide de $\mathrm{JL}$ et de $\mathrm{JC}$ (un mois de $\mathrm{JL} /$ un mois de $\mathrm{JC}$ ), appliquée à des béliers Ile-de-France ou Suffolk à partir de janvier (période pendant laquelle le poids testiculaire est minimal, voisin de $200 \mathrm{~g}$ ), entraîne, dans les 4-5 mois qui suivent, la reprise de croissance du poids testiculaire, puis son maintien à un niveau de saison sexuelle (proche de $300 \mathrm{~g}$ ) pendant au moins 2 ans (en fait toute la durée de l'expérimentation) (fig 5). II est possible d'utiliser l'alternance de $\mathrm{JL}$ et de JC ou celle d'un mois de flash et d'un mois de JC (Pelletier et Almeida, 1987). Des béliers lle-de-France ainsi traités (fig 6) et dont la semence est récoltée pendant l'habituelle contre-saison, produisent beaucoup plus de spermatozoïdes que des béliers non traités et d'une qualité équivalente à ceux récoltés en saison sexuelle (Chemineau et al, 1988).

Ce schéma a été testé sur une plus large échelle par un important Centre d'IA qui a soumis la moitié de son cheptel de béliers à une telle alternance. Les résultats obtenus montrent une importante augmentation de la production spermatique par rapport aux béliers témoins (tableau III).

\section{Chez le bouc (Delgadilio et al, 1991, 1992; Delgadillo et Chemineau, 1992)}

Le succès obtenu dans l'espèce ovine permettait d'envisager le passage dans l'espèce caprine, pour laquelle l'important saisonnement de la spermatogénèse conduit les centres d'IA à ne collecter les animaux que de septembre à février. Dans cette espèce, par ailleurs, l'augmentation du 
(a)

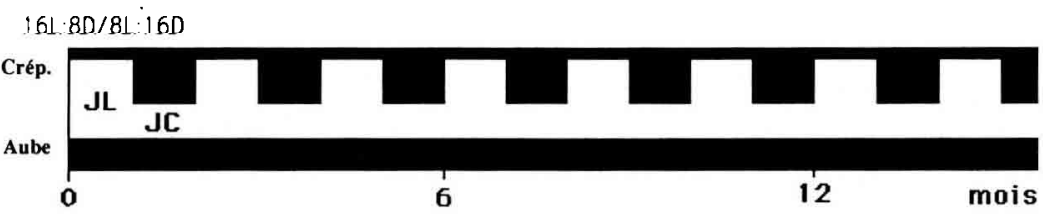

(b)

$71 \cdot 8 \mathrm{D}: 11 \cdot 8 \mathrm{D} / 81 \cdot 16 \mathrm{D}$

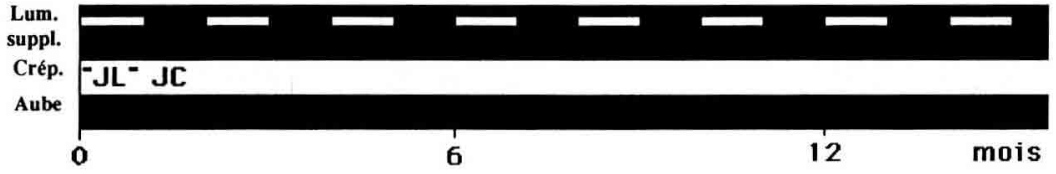

Poids testiculaire $(g)$
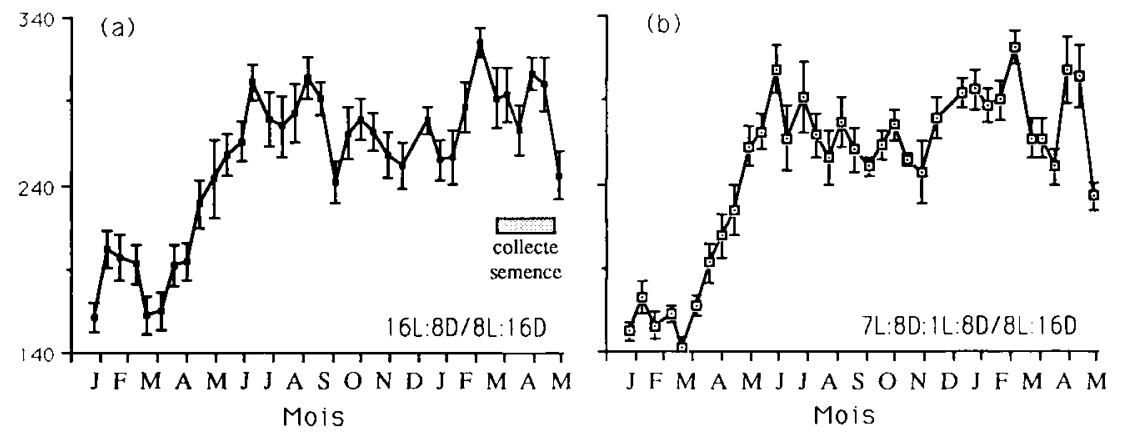

Fig 5. Poids testiculaire de béliers lle de France traités par une alternance rapide de jours long et de jours courts. a : 16L:8D/8L:16D) ou de "jours longs" et de jours courts. b : 7L:8D:1L:8D/8L:16D) (d'après Pelletier et Almeida, 1987).

nombre de doses d'IA stockées par bouc était un objectif économiquement intéressant. De janvier 1987 à mai 1990, 2 lots de 6 boucs ont été soumis à l'alternance d'1 mois de $\mathrm{JL}$ et d'1 mois de JC (soit une période de 2 mois) ou à l'alternance de 2 mois de $\mathrm{JL}$ et de 2 mois de JC (soit une période de 4 mois), en comparaison avec un troisième lot, témoin contemporain, soumis aux variations naturelles de la photopériode.
Comme chez les béliers, après $3-5$ mois selon les lots, le poids testiculaire augmente indiquant la reprise d'une activité spermatogénétique intense, puis il se maintient, au contraire de ce qui est observé chez les témoins, à un niveau élevé pendant le reste de l'expérience, soit une durée totale d'activité spermatogénétique élevée au moins égale à 3 années consécutives. Les différentes mesures réalisées sur les animaux traités montrent qu'ils maintiennent une acti- 

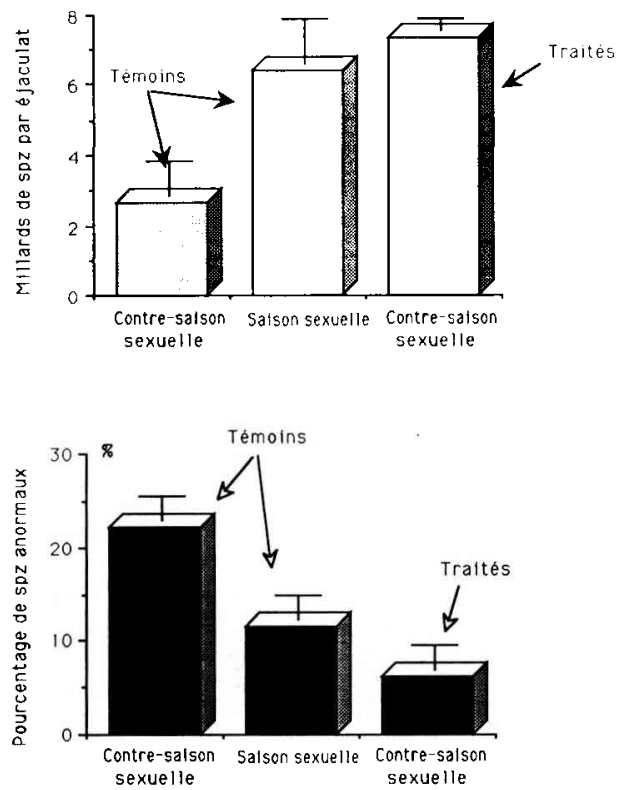

Fig 6. Production spermatique quantitative et qualitative de béliers lle de France traités ou non avec une alternance rapide de jours longs et de jours courts (pour la période de collecte, voir figure 5; d'après Almeida et al, cités par Chemineau et al, 1988).

vité sexuelle élevée et pratiquement constante : comportement sexuel, volume et concentration des éjaculats, nombre de spermatozoïdes, aptitude de ces derniers à supporter la congélation, etc.. sont du même niveau, voire supérieurs pour certains critères, à ceux rencontrés pendant la saison sexuelle annuelle. Le nombre total de spermatozoïdes produits par les boucs traités est toujours très supérieur à celui des boucs témoins et au total, le nombre de doses produites, utilisables pour I'I.A. est supérieur de $69 \%$ (période 2 mois) et $55 \%$ (période 4 mois) à celui du témoin.
La fertilité de la semence produite par les boucs traités n'est pas différente de celle produite par les boucs témoins pendant la saison sexuelle, ainsi que le montrent les 2 larges campagnes d'IA faites en 1988 et 1989 sur un grand nombre de femelles.

\section{Utilisation de la mélatonine seule : avance de la date de la saison sexuelle annuelle}

L'utilisation d'un traitement de mélatonine seul (sans traitement photopériodique préalable) a fait l'objet de nombreuses expérimentations notamment en Australie, Nouvelle Zélande, Grande Bretagne et France (Chemineau et al, 1991).

Chez les races peu saisonnées telles que le Mérinos, il permet une augmentation significative de la fertilité et de la prolificité, quelle que soit la date à laquelle il est employé. Chez les races saisonnées originaires de l'Europe du Nord, dont le début de saison se situe en septembre, ce type de traitement permet d'avancer de 11,5 mois le début de la saison sexuelle (Arendt et al, 1983; Symons et al, 1987). Dans ces races, le traitement n'est efficace que s'il commence à partir de la fin mai.

Plusieurs formes de distribution de la mélatonine ont été essayées, (distribution quotidienne par injection ou ingestion, implants sous cutanés, bolus intra-ruminal). Les implants sous cutanés, produisent également une augmentation du taux d'ovulation qui conduit à un accroissement de la prolificité.

Dans les races françaises, Limousine et Caussenarde du Lot, un tel traitement, employé avec insertion des implants (Mélovine $^{\mathrm{TM} *}$ ) 30-40 j avant l'introduction des

\footnotetext{
* Mélovine ${ }^{\mathrm{TM}}$ est une marque déposée, fabriquée par Hoecht, UK. Mélovine ${ }^{\mathrm{TM}}$ est aussi connu
} comme Regulin ${ }^{\circledR}$ dans d'autres pays. 
Tableau III. Utilisation d'un rythme photopériodique accéléré (un mois de jours longs alternant avec un mois de jours courts) dans des conditions de Centre d'IA (Intersélection, L'Aigle).

\begin{tabular}{|c|c|c|c|c|c|}
\hline \multirow[b]{2}{*}{ Race } & \multicolumn{2}{|c|}{ Nombre de béliers } & \multicolumn{3}{|c|}{ Supériorité par rapport au témoin (\%) } \\
\hline & Témoins & Traites & $\begin{array}{l}\text { Ejaculats } \\
\text { utilisables }\end{array}$ & $\begin{array}{c}\text { par } \\
\text { ejaculat }\end{array}$ & $\begin{array}{c}N b \text { total } \\
\text { spermatozoïdes } \\
\text { produits }\end{array}$ \\
\hline Rouge de l'Ouest & 5 & 6 & +5 & +20 & +26 \\
\hline lle de France & 4 & 4 & +22 & +27 & +55 \\
\hline Suffolk & 4 & 5 & +115 & +81 & +290 \\
\hline Charollais & 4 & 10 & +60 & +93 & +209 \\
\hline Total & 17 & 25 & +50 & +55 & +145 \\
\hline
\end{tabular}

Pezavent $P$ et al (résultats non publiés).

béliers pour la lutte naturelle, provoque un déclenchement de l'activité sexuelle en avance de saison et une augmentation significative de fertilité et de prolificité, aboutissant à un accroissement de $20 \%$ de la fécondité des brebis traitées (tableau IV). L'augmentation de prolificité induite par le traitement porte sur un accroissement significatif de la proportion d'agneaux nés doubles, mais pas de celle d'agneaux nés triples (fig 7).

Cette distribution de mélatonine sous forme d'implants est certainement la forme la plus aisée et la plus économique. $\mathrm{Ce}$ type de traitement est déjà commercialisé en Australie et devrait maintenant l'être assez rapidement en France et dans la CEE.

Tableau IV. Fertilité, prolificité et fécondité de brebis caussenardes du Lot et limousines, témoins ou traitées avec la Mélatonine et luttées naturellement. L'expérience s'est déroulée dans 9 troupeaux privés et les mâles ont été introduits pour la lutte, de fin mars à mi juin, 30-40 j après l'insertion d' 1 ou 2 implants de mélatonine (Mélovine ${ }^{\mathrm{TM}}$ ). Chemineau et al 1991.

\begin{tabular}{|c|c|c|c|}
\hline & $\begin{array}{c}\text { Fertilité } \\
(\%) \\
\text { (nombre de brebis) }\end{array}$ & $\begin{array}{c}\text { Prolificité } \\
\text { (nombre d'agneaux } \\
\text { par mise bas) }\end{array}$ & $\begin{array}{c}\text { Fécondité } \\
\text { (nombre d'agneaux } \\
\text { par brebis mise en lutte) }\end{array}$ \\
\hline Brebis Témoins & $\begin{array}{c}76 \\
(401)\end{array}$ & 1.35 & 1.02 \\
\hline $\begin{array}{l}\text { Brebis traitées } \\
\text { avec la Mélatonine }\end{array}$ & $\begin{array}{c}85 \\
(447)\end{array}$ & 1.42 & 1.21 \\
\hline
\end{tabular}




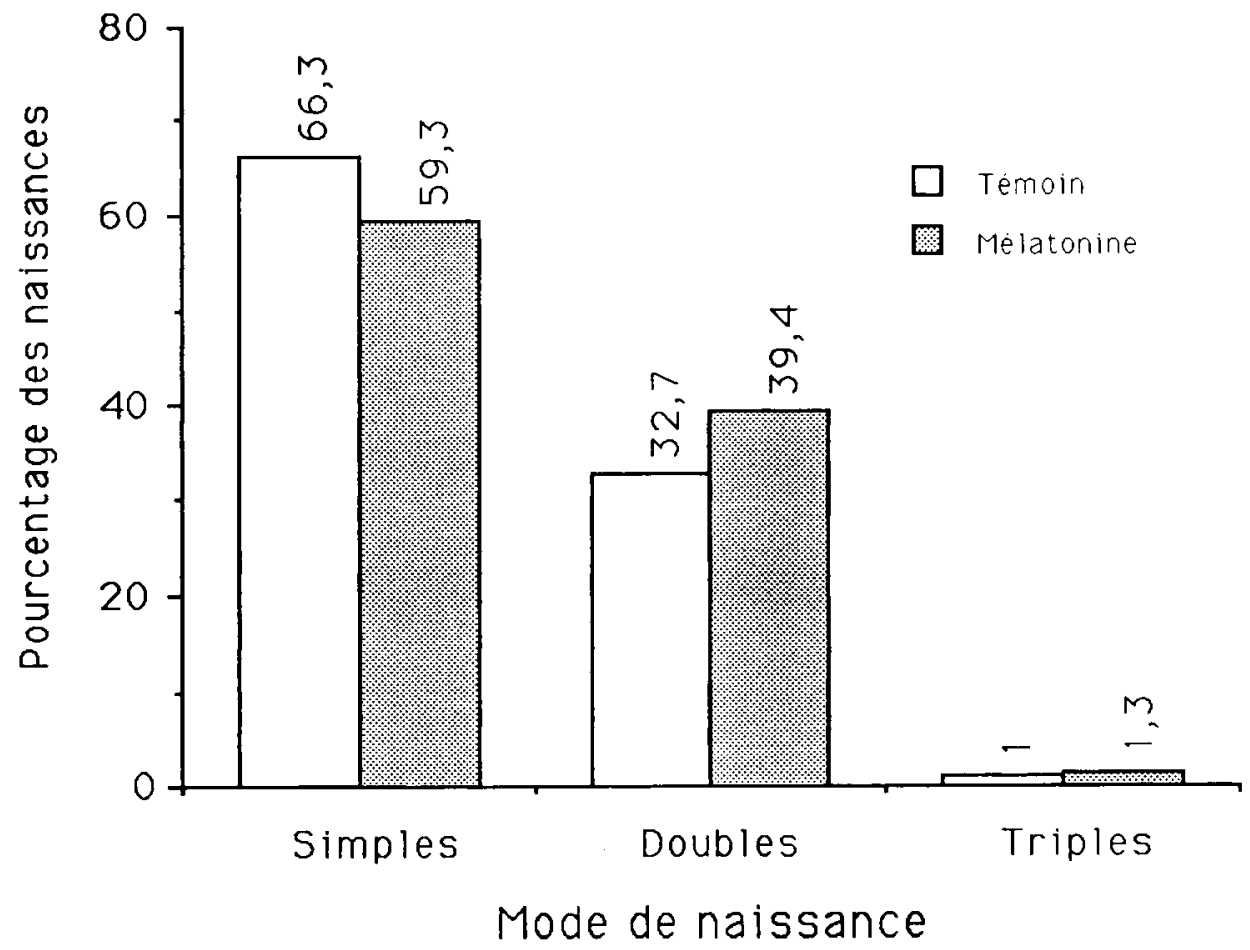

Fig 7. Répartition des naissances selon leur mode après lutte naturelle, chez des brebis témoins et des brebis traitées avec de la mélatonine (Mélovine; d'après Chemineau et al, 1991).

\section{CONCLUSION}

Dans les conditions climatiques tempérées, les variations de la durée d'éclairement sont les principales responsables des variations saisonnières de reproduction des ovins et caprins.

L'abolition totale de ces variations chez les mâles de ces 2 dernières espèces grâce à l'utilisation d'une alternance rapide de $\mathrm{JL}$ et de JC permet d'augmenter très sensiblement la production spermatique et, par là, d'accroître le nombre de doses d'IA utilisables par mâle amélioré. Par ailleurs, chez la femelle comme chez le mâle, les résultats obtenus jusqu'à présent avec le traitement utilisant flash lumineux puis la mélatonine permettent d'envisager une maîtrise efficace de la reproduction à contre-saison. Un tel traitement aboutirait à une fertilité et une prolificité équivalentes à celles de la saison sexuelle. L'utilisation de la mélatonine seule, permet un déclenchement plus précoce de la saison sexuelle des brebis et une augmentation importante de la fécondité de celles-ci.

\section{REMERCIEMENTS}

Les auteurs tiennent à remercier $D$ Chesneau, B Leboeuf (INRA), B Escot (EDE d'Indre et Loire), G Brice, C Jardon (ITOVIC), JP Vaur, H 
Issaly (EDE du Lot), JP Belloc (OVITEST), M Briois (Confédération des producteurs de lait de brebis et de fromage de Roquefort), LD Staples (Applied Biotechnologies). Les expérimentations ont été financées partiellement par EDF, la région Poitou-Charentes et la région Centre, Sanofi santé animale, CAMCO et le Critt-ISIS.

\section{RÉFÉRENCES}

Arendt J, Symons AM, Laud CA, Pryde SJ (1983) Melatonin can induce early onset of the breeding season in ewes. $J$ Endocrinol $97,395-400$

Chemineau $P$ (1989) Le désaisonnement des chèvres par la lumière et la mélatonine. Chèvre 171, 18-22

Chemineau $P$, Normant E, Ravault JP, Thimonier J (1986) Induction and persistence of pituitary and ovarian activity in the out-ofseason lactating dairy goat after a treatment combining a skeleton photoperiod, melatonin and the male effect. $J$ Reprod Fertil 78, 497504

Chemineau P, Pelletier J, Guérin $Y$, Colas $G$, Ravault JP, Touré G, Almeida G, Thimonier J, Ortavant R (1988) Photoperiodic and melatonin treatments for the control of seasonal reproduction in sheep and goats. Reprod Nutr Dév 28, 409-422

Chemineau P, Delgadillo JA, Malpaux B, Pelletier J (1989) Annual clock and control of domestic mammal reproduction. Perspect Androl 53, 307-315

Chemineau P, Vandaele E, Brice G, Jardon C (1991) Utilisation des implants de mélatonine pour l'amélioration des performances de reproduction chez la brebis. Recl Méd Vét Alfort $167(3 / 4), 227-239$

Colas G, Guérin Y, Briois M, Ortavant R (1987) Photoperiodic control of testicular growth in the ram lamb. Anim Reprod Sci 13, 255-262

Delgadillo JA, Chemineau P (1992) Abolition of the seasonal release of luteinizing hormone and testosterone in Alpine male goats (Capra hircus) by short photoperiodic cycles. $J$ Reprod Fertil 94, 45-55

Delgadillo JA, Leboeuf $B$, Chemineau $P$ (1991) Decrease in the seasonality of sexual behavior and sperm production in bucks by ex- posure to short photoperiodic cycles. Theriogenology 36(5), 755-770

Delgadillo JA, Leboeuf B, Chemineau P (1992) Abolition of seasonal variations in semen quality and maintenance of sperm fertilizing ability by photoperiodic cycles in goat bucks. Small Rumin Res 6 (sous presse)

Howles CM, Webster GM, Haynes NB (1980) The effect of rearing under a long or short photoperiod on testis growth, plasma testosterone and prolactin concentrations, and the development of sexual behaviour in rams. $J$ Reprod Fertil 60, 437-447

Kennaway DJ, Hooley RD, Seamark RF (1980) Effects of melatonin feeding on serum prolactin content and the onset of oestrous activity in goats. Aust Soc Reprod Biol 12, (Abstr)

Kennaway DJ, Gilmore TA, Seamark RF (1982) Effect of melatonin feeding on serum prolactin and gonadotropin levels and the onset of seasonal oestrus cyclicity in sheep. Endocrinology 110, 1766-1772

Malpaux B, Robinson JE, Wayne NL, Karsch FJ (1989) Regulation of the onset of the breeding season of the ewe: importance of long days and of an endogenous reproductive rhythm. J Endocrinology 122, 269-278

Malpaux B, Daveau A, Gayrard V, Thiéry JC (1990) Melatonin acts in the medial basal hypothalamus to control reproduction in the ewe. Soc Res Biol Rhythms Charlottesville USA, abstr 41

Ortavant $R$ (1977) Photoperiodic regulation of reproduction in the sheep. In: Symp management of reproduction in sheep and goats, Madison (WI, USA), 24-25 juillet, 58-71

Ortavant R, Pelletier J, Ravault JP, Thimonier J, Volland-Nail P (1985) Photoperiod: main proximal and distal factor of the circannual cycle of reproduction in farm mammals. In: Oxford Rev reprod Biol, Clarendon Press, Oxford, 7, 305-345

Pelletier J, Almeida G (1987) Short light cycles induce persistent reproductive activity in lle-deFrance rams. J Reprod Fertil suppl 34, 215-226

Pelletier J, Thimonier J (1987) The measurement of daylength in the Ile-de-France ram. $J$ Reprod Fertil 81, 181-186

Pelletier J, Blanc M, Daveau A, Garnier DH, Ortavant $R$, de Reviers MM, Terqui M (1981) Mechanisms of light action in the ram: a pho- 
tosensitive phase for $\mathrm{LH}, \mathrm{FSH}$, testosterone and testis weight? In: Photoperiodism and reproduction. Nouzilly, France, 24-25 September (R Ortavant, J Pelletier, JP Ravault, eds) INRA, Paris, 117-134

de Reviers MM, Ravault JP, Tillet $Y$, Pelletier J (1989) Melatonin binding sites in the sheep pars tuberalis. Neurosc Lett 100, 89-93

Robinson JE, Karsch FJ (1984) Refractoriness to inductive day lengths terminates the breeding season of the Suffolk ewe. Biol Reprod $31,656-663$
Robinson JE, Wayne N, Karsch FJ (1985) Refractoriness to inhibitory daylength initiates the breeding season of the Suffolk ewe. Biol Reprod 32, 1024-1030

Symons AM, Arendt J, Poulton AL, English J (1987) Induction of early seasonal sensitivity to melatonin in Suffolk-cross ewes. Chronobiol Int 4, 219-223

Thimonier J (1989) Contrôle photopériodique de l'activité ovulatoire chez la brebis. Existence de rythmes endogènes. Thèse Doctorat Univ Tours, $112 p$ 